Fayetteville State University

DigitalCommons@Fayetteville State University

Math and Computer Science Faculty Working

Papers

Math and Computer Science

$10-2015$

\title{
Mathematical Modeling and Simulations of the Pathophysiology of Type-2 Diabetes Mellitus
}

Frank K. Nani

Fayetteville State University, fnani@uncfsu.edu

Mingxian Jin

Fayetteville State University, mjin@uncfsu.edu

Follow this and additional works at: https://digitalcommons.uncfsu.edu/macsc_wp

Part of the Medical Biomathematics and Biometrics Commons

\section{Recommended Citation}

Nani, Frank K. and Jin, Mingxian, "Mathematical Modeling and Simulations of the Pathophysiology of Type-2 Diabetes Mellitus" (2015). Math and Computer Science Faculty Working Papers. 25.

https://digitalcommons.uncfsu.edu/macsc_wp/25

This Conference Proceeding is brought to you for free and open access by the Math and Computer Science at DigitalCommons@Fayetteville State University. It has been accepted for inclusion in Math and Computer Science Faculty Working Papers by an authorized administrator of DigitalCommons@Fayetteville State University. For more information, please contact dballar5@uncfsu.edu. 


\title{
Mathematical Modeling and Simulations of the Pathophysiology of Type-2 Diabetes Mellitus
}

\author{
Frank Nani and Mingxian Jin \\ Department of Mathematics and Computer Science \\ Fayetteville State University \\ Fayetteville, NC 28301, USA
}

\begin{abstract}
The pathophysiology of Type 2 Diabetes Mellitus (T2DM) is modelled using a coupled system of non-linear deterministic differential equations. An attempt is made to construct to a clinically plausible mathematical model that incorporates the homeostasis associated with endocrinological regulation of glucose and glycogen levels in the human body, by the hormones, insulin and glucagon. The model variables include the concentrations of glucose in the venous blood plasma, the concentration of glycogen in the liver/tissues, the concentration of the hormone glucagon, and the concentration of insulin in the venous blood plasma. The physiological interactions between the model parameters are depicted by clinically measurable rate constants and biophysically quantifiable stoichiometric coefficients. The processes of gluconeogenesis, glycogenolysis, and pulsatile insulin secretion during type 2 diabetes are modelled using plausible auxiliary functions. Investigative computer simulations are performed to elucidate various hypothetical scenarios of glycemia, patho-physiology of T2DM and insulinoma associated hypoglycemia which results from excessive insulin production probably due to a tumor. This study has demonstrated the necessity of simultaneous monitoring of plasma glucose, glucagon, insulin, and glycogen levels in the proper assessment of the pathophysiology of type 2 diabetes and during determination of the therapeutic efficacy of anti-diabetic drugs.
\end{abstract}

Keywords--Mathematical modeling; diabetes mellitus; dynamics of glucose; glycogen; glucagon; insulin

\section{INTRODUCTION}

Type 2 diabetes mellitus (T2DM) is characterized by insulin resistance, insulin hypo-secretion to $\beta$ cell impairment and/or over production of glucose. A person is diagnosed as diabetic if the fasting venous plasma glucose concentration is greater than $126 \mathrm{mg} / \mathrm{dL}$ and/or the 2-hour post-glucose load of $75 \mathrm{~g}$ of anhydrous glucose yield a venous plasma glucose concentration of $200 \mathrm{mg} / \mathrm{dL}$ [1]. Alternative novel tests for diagnosis include the use of glycated hemoglobin $\left(\mathrm{Hb}_{\mathrm{AlC}}\right)$ with suggested levels of about $6.5 \%$ [2].

The pre-disposing historical high risk factors for T2DM include increasing age (old age), central obesity, dietary polyphagia of animal fats, carbonated drinks, lack of physical exercises, familial genetic factors, history of gestational diabetes, polycystic ovary syndrome, and severe mental illness, presence of hypertension, hyperlipidemia, ethnicity and cardio-metabolic factors. Recent emerging risk factors include sleep deprivation, drug-induced metabolic changes, environmental pollutants, low birth weight and fetal malnutrition $[3,4,5,6,7]$.

Many mathematical models in the literature were devoted mainly to the dynamics of glucose in relation to the hormone, insulin. The basic model was proposed by Bolie [9], who used the following system of ordinary differential equations

$$
\begin{aligned}
& \frac{d G}{d t}=-a_{1} G-a_{2} I+P \\
& \frac{d I}{d t}=-a_{3} G-a_{4} I
\end{aligned}
$$

where $G=G(t)$ represents glucose concentration, $I=I(t)$ represents insulin concentration, and $P, a_{1}, a_{2}, a_{3}, a_{4}$ are model parameters.

A clinically relevant minimal model of glucose-insulin dynamics was proposed by Bergman, Bowden, and Cobelli in [10]. De Gaetano Arino [11] constructed a dynamical model of insulin-glucose dynamics using an aggregated integrodifferential delay equations. Several other models were constructed by the following authors: Li et. al. [12], Cobelli and Tomaseth [13], Lam et. al. [14], Mari [8].

The main objective of the research presented in this paper is to construct a clinically plausible model that depicts the homeostasis of glucose-insulin regulatory dynamics and the pathophysiology of T2DM.

\section{DEFinitions of Model Parameters}

The explicit non-linear deterministic mathematical equations describing the patho-physiology of T2DM are presented in this section.

$x_{1}(t)$ : the concentration of glucose in the blood plasma of the patient at any time $t$ during T2DM.

$x_{2}(t)$ : the concentration of glycogen in the blood plasma of the patient at any time $t$ during T2DM.

$x_{3}(t)$ : the concentration of glucagon in the blood plasma of the patient at any time $t$ during T2DM. 
$x_{4}(t)$ : the concentration of insulin in the blood plasma of the patient at any time $t$ during T2DM.

$G_{i}\left(x_{i}\right)$ : the rate constant depicting exogenous input into the $i^{\text {th }}$ compartment by either post-prandial uptake or intravenous (i.v.) infusion or by subcutaneous injection $[i=1,2,3,4]$.

$S_{1}\left(x_{3}, x_{4}\right)$ : the rate of hepatic glucose synthesis by gluconeogenesis

$S_{2}\left(x_{3}, x_{4}\right)$ : the rate of glycogen bio-synthesis.

$S_{3}\left(x_{1}, x_{2}\right)$ : the rate of glucagon bio-synthesis in alpha cells of the pancreas.

$S_{4}\left(x_{1}, x_{4}\right)$ : the rate of insulin bio-synthesis in beta cells of the pancreas.

In particular, the following homeostasis functions are used:

$$
\begin{aligned}
& S_{1}\left(x_{3}, x_{4}\right)=\frac{c_{1} x_{3}}{1+\mu_{1} x_{4}} \\
& S_{2}\left(x_{3}, x_{4}\right)=\frac{c_{2} x_{4}}{1+\mu_{2} x_{3}} \\
& S_{3}\left(x_{1}, x_{2}\right)=\frac{c_{3} x_{2}}{1+\mu_{3} x_{1}} \\
& S_{4}\left(x_{1}, x_{2}\right)=\frac{c_{4} x_{1}}{1+\mu_{4} x_{2}}
\end{aligned}
$$

$k_{i}$ : the rate constant depicting catabolic degradation $[i=1,2,3,4]$ pertaining to exponential decay kinetics.

$k_{0 i}$ : the rate constant depicting catabolic degradation $[i=1,2,3,4]$ pertaining to linear decay kinetics.

$\left(a_{14}, a_{41}\right)$ : stoichiometric kinetic constant involved in the action of insulin $\left(x_{4}\right)$ on glucose $\left(x_{1}\right)$.

$\sigma_{2}:$ stoichiometric kinetic constant involved in the production of glycogen.

$\left(a_{23}, a_{32}\right)$ : stoichiometric kinetic constant involved in the action of glucagon $\left(x_{3}\right)$ on glycogen $\left(x_{2}\right)$.

$\sigma_{1}:$ stoichiometric kinetic constant involved in the production of glucose.

$f_{i}(t)=|\lceil\sin n t\rceil|$ represents pulsatile input function for each $i$ $[i=1,2,3,4]$.

\section{Model Equations For TyPe-2 Diabetes Mellitus}

In this section, an elaborate physiological description and mathematical formulation of the model equations are presented.

\section{A. The Glucose Dynamics Equation}

The rate of change of glucose in the body is equal to the post prandial input $\left(G_{1} f_{1}(t)\right)$, plus the rate of glucose production by gluconeogenesis $\left(S_{1}\left(x_{3}, x_{4}\right)\right)$ which is breakdown of pyruvate into glucose mediated by insulin and glucagon, plus the rate of glucose production by glycogenolysis involving action of glucagon on glycogen $\left(\sigma_{1} x_{2} x_{3}\right)$, minus the rate of loss of glucose during glycogenesis due to action of insulin on glucose $\left(a_{14} x_{1} x_{4}\right)$, minus the rate of catabolism of glucose during glycolysis $\left(k_{1} x_{1}\right)$, minus the linear rate of degradation of glucose by utilization during exercise and physiologic activity in the brain, skeletal muscles and cardiac muscles $\left(k_{01}\right)$. The glucose dynamics equation is shown as below:

$$
\frac{d x_{1}}{d t}=G_{1} f_{1}(t)+S_{1}\left(x_{3}, x_{4}\right)+\sigma_{1} x_{2} x_{3}-a_{14} x_{1} x_{4}-k_{1} x_{1}-k_{01}
$$

\section{B. The Glycogen Dynamics Equation}

Glycogen dynamical equation depends on the postprandial rate of glycogen input $G_{2} f_{2}(t)$; the bio-synthesis of glycogen term $S_{2}\left(x_{3}, x_{4}\right)$ which is mediated by glucagon and insulin and stored in body tissues including muscle; the rate of de novo synthesis of glycogen due to action of insulin on glucose $\left(\sigma_{2} x_{1} x_{4}\right)$ and mediated by glycogen synthase; the rate of catabolism of glycogen by glycogenolysis in liver ( $\left.-a_{23} x_{2} x_{3}\right)$; rate of loss of glycogen by enzymatic/exponential degradation mediated by glycogen phosphorylase $\left(-k_{2} x_{2}\right)$; and rate of linear degradation of glycogen $\left(-k_{02}\right)$. The catabolic breakdown of glycogen is mediated primarily by glucagon but there are other hormones that are involved. These include cortisol, somatotropin, and catecholamines. The glycogen dynamical equation thus takes the form:

$\frac{d x_{2}}{d t}=G_{2} f_{2}(t)+S_{2}\left(x_{3}, x_{4}\right)+\sigma_{2} x_{1} x_{4}-a_{23} x_{2} x_{3}-k_{2} x_{2}-k_{02}$

\section{The Glucagon Dynamics Equation}

The rate of change of glucagon in the body equals to the rate of exogenous input $\left(G_{3} f_{3}(t)\right)$ by intravenous or intramuscular injection, plus rate of biosynthesis of glucagon and glycogen $\left(S_{3}\left(x_{1}, x_{2}\right)\right)$ mediated by the levels of glucose and glycogen, minus the rate of loss of glucagon during glycogenolysis $\left(a_{32} x_{2} x_{3}\right)$ in the liver, minus degradation of glucagon enzymatically $\left(k_{3} x_{3}\right)$, and minus linear catabolism of glucagon $\left(k_{03}\right)$. The glucagon dynamical equation thus takes the following form: 
$\frac{d x_{3}}{d t}=G_{3} f_{3}(t)+S_{3}\left(x_{1}, x_{2}\right)-a_{32} x_{2} x_{3}-k_{3} x_{3}-k_{03}$

\section{The Insulin Dynamics Equation}

The rate of change of insulin in the venous blood plasma is equal to the rate of exogenous input $\left(G_{4} f_{4}(t)\right)$ by intravenous or sub-cutaneous injection, plus the bio-synthesis of insulin in the $\beta$-cells of the islets of Langerhans in the pancreas mediated by the relative concentrations of glucose and glycogen $\left(\left(S_{4}\left(x_{1}, x_{2}\right)\right)\right.$, minus the rate of insulin catabolism during the mediation of conversion of glucose to glycogen $\left(a_{41}\right.$ $\left.x_{1} x_{4}\right)$, minus the exponential degradation of insulin $\left(k_{4} x_{4}\right)$, minus the linear degradation of insulin $\left(k_{03}\right)$. Thus the insulin dynamical equation takes the form:

$\frac{d x_{4}}{d t}=G_{4} f_{4}(t)+S_{4}\left(x_{1}, x_{2}\right)-a_{41} x_{1} x_{4}-k_{4} x_{4}-k_{04}$

\section{COMPUTER Simulation Results AND Discussion}

In this section, investigative computer simulations are performed to elucidate some aspects of T2DM pathophysiology using hypothetical clinical parametric configurations. For all the simulations, the pulsatile input function $f_{i}(t)$ is defined by the formula:

$$
f_{i}(t)=|\lceil\sin n t\rceil| \text { where } n=24
$$

The time scale for all simulations is hours. The values of rate constants are all estimated and are hypothetical. The units for variables $x_{1}, x_{2}, x_{3}$, and $x_{4}$ are as follow: $x_{1}(m g / d L), x_{2}(\mathrm{~g})$, $x_{3}(p g / m L)$, and $x_{4}(\mu l U / m L)$.

\section{A. Hypothetical Patient \#1}

TABle I. Parametric Configuration $P_{1}$

\begin{tabular}{|l|l|l|l|}
\hline$G_{1}=90.0$ & $G_{2}=20$ & $G_{3}=20$ & $G_{4}=50$ \\
$c_{1}=0.25$ & $c_{2}=0.975$ & $c_{3}=10.5$ & $c_{4}=25.0$ \\
$\mu_{1}=0.8$ & $\mu_{2}=0.7$ & $\mu_{3}=0.009$ & $\mu_{4}=0.085$ \\
$\sigma_{1}=0.25$ & $\sigma_{2}=0.35$ & $a_{32}=0.1$ & $a_{41}=0.15$ \\
$k_{1}=2.85$ & $a_{23}=0.1$ & $k_{3}=0.00001$ & $k_{4}=0.0001$ \\
$a_{14}=0.50$ & $k_{2}=0.95$ & $k_{03}=0.00015$ & $k_{04}=0.0025$ \\
$k_{01}=10.50$ & $k_{02}=0.75$ & $x_{30}=100$ & $x_{40}=20$ \\
$x_{10}=90$ & $x_{20}=80$ & & \\
\hline
\end{tabular}
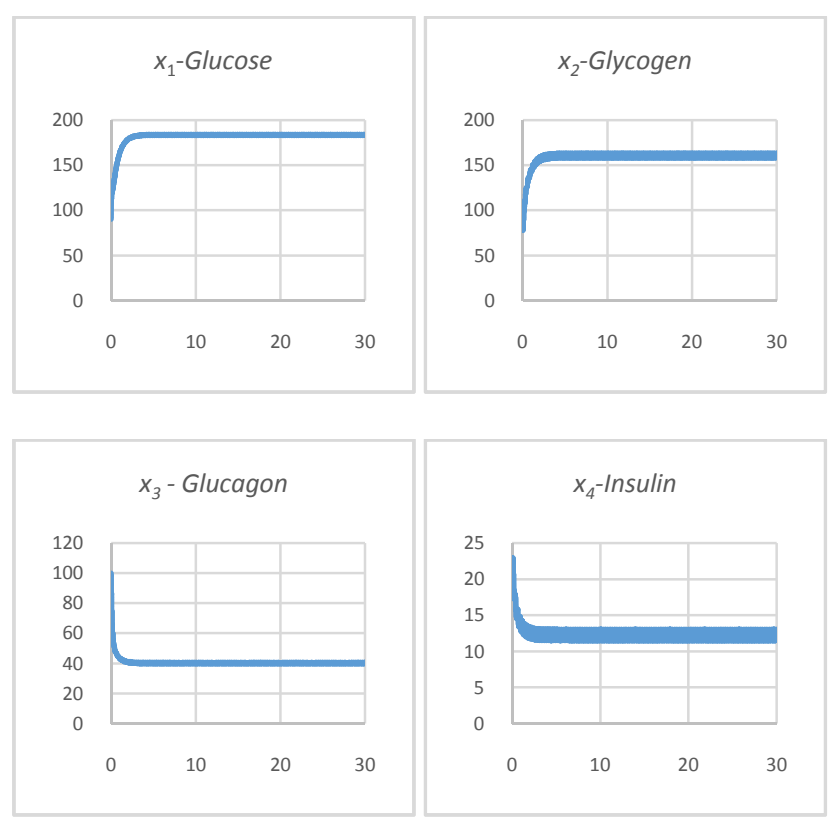

Figure 1. Simulation results using parametric configuration $P_{1}$

As shown in Figure 1, the dynamics of glucose, glycogen, glucagon, and insulin in hypothetical patient \#1 seems normal. In particular, the steady status values are approximately $180 \mathrm{mg} / \mathrm{dL}$ for $x_{1}, 160 \mathrm{~g}$ for $x_{2}, 40 \mathrm{pg} / \mathrm{mL}$ for $x_{3}$, and $10 \sim 15 \mathrm{mIU} / \mathrm{mL}$ for $x_{4}$. Compared with the standard ranges, the patient's glucose, glycogen, glucagon and insulin levels are regulated by feedback homeostasis.

\section{B. Hypothetical Patient \#2}

TABle II. PARAmetric Configuration $P_{2}$

\begin{tabular}{|l|l|l|l|}
\hline$G_{1}=90.0$ & $G_{2}=20$ & $G_{3}=20$ & $G_{4}=50$ \\
$c_{1}=0.25$ & $c_{2}=0.975$ & $c_{3}=3.75$ & $c_{4}=3.85$ \\
$\mu_{1}=1$ & $\mu_{2}=1$ & $\mu_{3}=1$ & $\mu_{4}=1$ \\
$\sigma_{1}=0.55$ & $\sigma_{2}=0.45$ & $a_{32}=0.0175$ & $a_{41}=0.09$ \\
$k_{1}=1.5$ & $a_{23}=0.5$ & $k_{3}=0.001$ & $k_{4}=0.0001$ \\
$a_{14}=0.50$ & $k_{2}=0.95$ & $k_{03}=0.0015$ & $k_{04}=0.0025$ \\
$k_{01}=7.50$ & $k_{02}=0.75$ & $x_{30}=100$ & $x_{40}=20$ \\
$x_{10}=90$ & $x_{20}=80$ & & \\
\hline
\end{tabular}



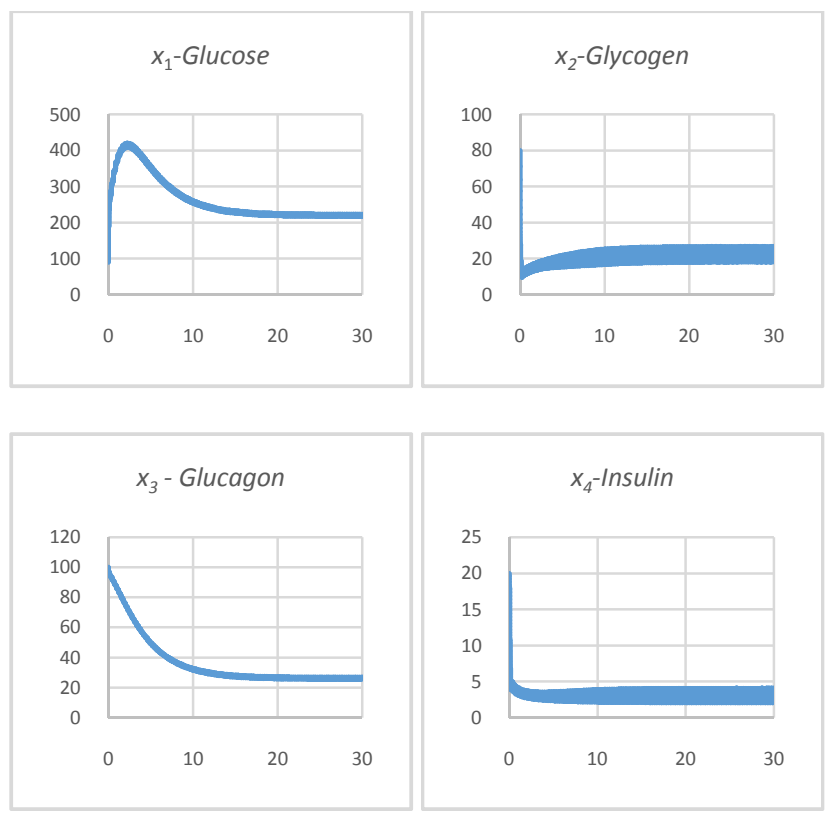

Figure 2. Simulation results using parametric configuration $P_{2}$

An inspection of the dynamics of glucose, glycogen, glucagon and insulin in hypothetical patient \#2 shown in Figure 2 reveals that this patient has hyperglycemia and low insulin levels. Thus the patient is either pre-diabetic or diabetic.

\section{Hypothetical Patient \#3}

TAble III. Parametric Configuration $P_{3}$

\begin{tabular}{|l|l|l|l|}
\hline$G_{1}=90.0$ & $G_{2}=20$ & $G_{3}=20$ & $G_{4}=50$ \\
$c_{1}=0.25$ & $c_{2}=0.975$ & $c_{3}=3.75$ & $c_{4}=3.85$ \\
$\mu_{1}=0.95$ & $\mu_{2}=0.85$ & $\mu_{3}=0.75$ & $\mu_{4}=0.70$ \\
$\sigma_{1}=0.55$ & $\sigma_{2}=0.45$ & $a_{32}=0.0175$ & $a_{41}=0.09$ \\
$k_{1}=1.5$ & $a_{23}=0.5$ & $k_{3}=0.001$ & $k_{4}=0.0001$ \\
$a_{14}=0.05$ & $k_{2}=0.95$ & $k_{03}=7.5$ & $k_{04}=0.0025$ \\
$k_{01}=7.50$ & $k_{02}=0.75$ & $x_{30}=100$ & $x_{40}=20$ \\
$x_{10}=90$ & $x_{20}=80$ & & \\
\hline
\end{tabular}
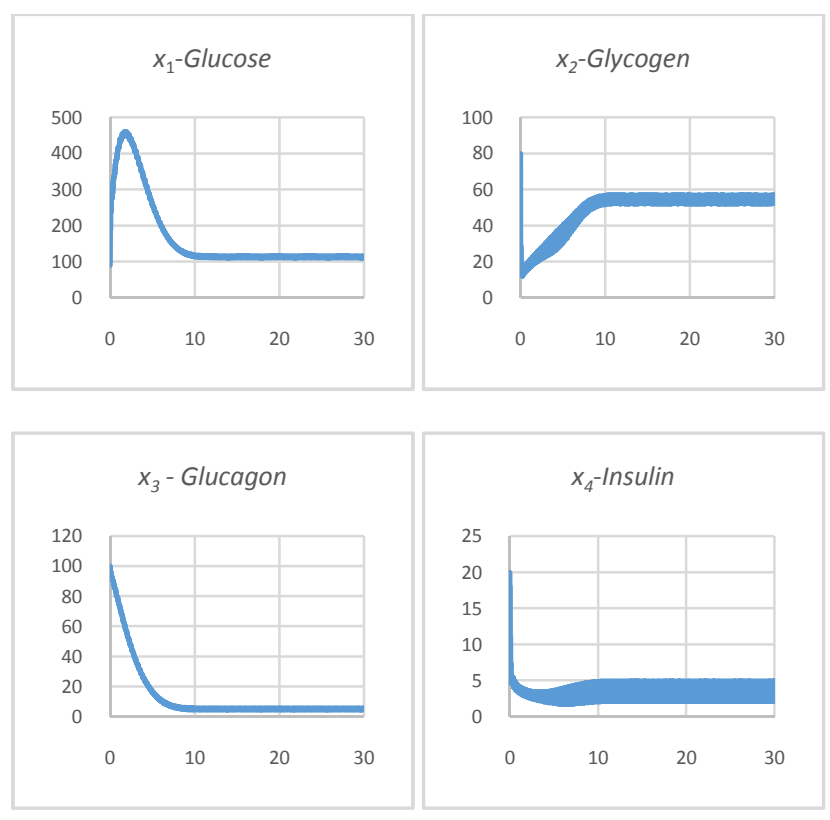

Figure 3. Simulation results using parametric configuration $P_{3}$

The glucose, glycogen, glucagon, and insulin profile of hypothetical patient \#3 shows that almost perfect homeostasis as shown in Figure 3. All the values are in the normal ranges. This this patient is free of diabetes.

\section{Hypothetical Patient \#4}

TABle IV. Parametric Configuration $P_{4}$

\begin{tabular}{|l|l|l|l|}
\hline$G_{1}=90.0$ & $G_{2}=20$ & $G_{3}=60$ & $G_{4}=50$ \\
$c_{1}=0.25$ & $c_{2}=0.975$ & $c_{3}=40$ & $c_{4}=25$ \\
$\mu_{1}=0.8$ & $\mu_{2}=0.7$ & $\mu_{3}=0.000009$ & $\mu_{4}=0.0085$ \\
$\sigma_{1}=0.25$ & $\sigma_{2}=0.35$ & $a_{32}=1.5$ & $a_{41}=0.09$ \\
$k_{1}=4.85$ & $a_{23}=0.1$ & $k_{3}=0.05$ & $k_{4}=0.00001$ \\
$a_{14}=0.5$ & $k_{2}=2.5$ & $k_{03}=0.15$ & $k_{04}=0.0025$ \\
$k_{01}=10.50$ & $k_{02}=0.75$ & $x_{30}=100$ & $x_{40}=60$ \\
$x_{10}=90$ & $x_{20}=80$ & & \\
\hline
\end{tabular}



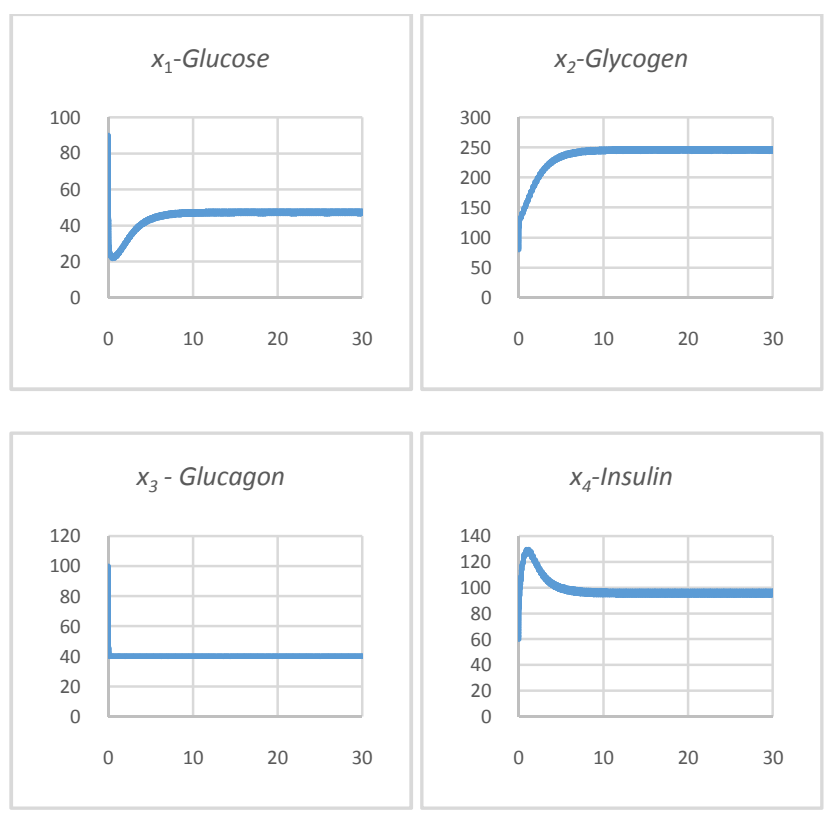

Figure 4. Simulation results using parametric configuration $P_{4}$

In Figure 4, the hypothetical patient \#4 exhibits the clinical manifestations of hypoglycemia which could be probably due to insulinoma that produces excessive levels of insulin that depletes the glucose levels by converting them into glycogen.

\section{CONCLUSION AND DISCUSSION}

This research shows the relative importance of mathematical modeling in diabetes research. The major hurdle is how to acquire clinically relevant data to validate the model and perform simulations. The use of Michaelis-Menten type functions make it extremely difficult to estimate the values of the rate constants. In order to solve this problem, hypothetical estimates were used. In the future, more realistic parametric estimates will be used. Nevertheless, this model is one of the first to attempt to describe the pathophysiology of Type 2 Diabetes Mellitus by incorporating most of the physiological aspects of the homeostasis of glucose, glycogen, glucagon and insulin. The computer simulations demonstrate the usefulness of the model equations in describing endocrinopathies associated with insulin and glucagon.

\section{ACKNOWLEDGMENT}

This research work is supported by a summer grant from the Graduate School at Fayetteville State University.

\section{REFERENCES}

[1] Bar RG, Nathan DM, Meigs JB, and Singer DE (2002). Tests of glycemia for the diagnosis of diabetes mellitus. Ann. Intern. Med. 137: 263-272.

[2] World Health Organization (WHO) Consultation (2006). Definition of diabetes mellitus and intermediate hyperglycemia. Geneva: WHO

[3] Jones OA, Maguire ML, Griffin JL (2008). Environmental pollution and diabetes: a neglected association. Lancet 371, 287288

[4] Ma RC, Kong AP, Chan N, Tong PC, Chan JC (2007). Druginduced endocrine and metabolic disorders. Drug Saf. 30: 215245

[5] Gangwisch JE, Heymsfield SB, et. al. (2007). Sllep duration as a risk factor for diabetes incidence in a large US sample. Sleep. 30: 1667-1673

[6] Ma R, Chan J (2009). Metabolic Complications of Obesity in: Williams G, Frubeck G, eds. Obesity: Science to Practice, John Wiley \& Sons Ltd. 235-270

[7] Eriksson J, Fransilla-Kallunki A, Ekstand A, et al. (1995). Early metabolic depicts in persons at risk of non-insulin dependent diabetes mellitus. N. Europ. Med. 321: 690-695

[8] Mari A: Mathematical modelling in glucose metabolism and insulin secretion. Current Opinion Clinical Nutrition Metabolism Care 2002, 5:495-501

[9] Bolie VW: Coefficients of normal blood glucose regulation. J Appl Physiol 1961, 16:783-788

[10] Bergman RN, Bowden CR, Cobelli C: The Minimal Model approach to quantification of factors controlling glucose disposal in man. In Carbohydrate Metabolism. Volume chap 13. Edited by Cobelli, Bergman. John Wiley \& Sons Ltd; 1981::269-293

[11] De Gaetano A, Arino O: A statistical approach to the determination of stability for dynamical systems modelling physiological processes. Math Comput Modelling 2000, 31:4151

[12] Li J, Kuang Y, Li B: Analysis of IVGTT Glucose-Insulin Interaction Models with time delay. Discrete and Continuous Dynamical Systems Series B 2000, 1(1):103-124

[13] Cobelli C, Thomaseth K: Optimal input design for identification of compartimental models : theory and applications to a model of glucose kinetics

[14] Lam ZH, Hwang KS, Lee JY, Chase JG, and Walker GC: Active insulin infusion using optimal and derivative weighted control. Medical engineering physics 2002, 24:663-672 